\title{
Mutations en Saxe - potentialités de développement et disparités régionales
}

Transition process in Saxony : potential developments and regional disparities Transformationsprozesse in Sachsen: Entwicklungspotentiale und regionale Disparitäten

\section{Helga Schmidt}

\section{(2) OpenEdition}

\section{Journals}

Édition électronique

URL : http://journals.openedition.org/rge/2844

DOI : $10.4000 /$ rge.2844

ISSN : 2108-6478

Éditeur

Association des géographes de l'Est

Édition imprimée

Date de publication : 1 juin 2001

ISSN : 0035-3213

Référence électronique

Helga Schmidt, « Mutations en Saxe - potentialités de développement et disparités régionales », Revue Géographique de l'Est [En ligne], vol. 41 / 3 | 2001, mis en ligne le 09 juillet 2013, consulté le 08 septembre 2020. URL : http://journals.openedition.org/rge/2844 ; DOI : https://doi.org/10.4000/rge. 2844

Ce document a été généré automatiquement le 8 septembre 2020

Tous droits réservés 


\title{
Mutations en Saxe - potentialités de développement et disparités régionales
}

\author{
Transition process in Saxony : potential developments and regional disparities \\ Transformationsprozesse in Sachsen: Entwicklungspotentiale und regionale \\ Disparitäten
}

Helga Schmidt

\section{Introduction}

1 Les changements politiques survenus en Allemagne depuis 1990 ont complètement bouleversé le cadre de l'évolution démographique, économique, sociale et écologique ainsi que l'organisation spatiale du land de Saxe (Freistaat Sachsen). Ce fut et c'est tout à la fois une chance et un défi. Dans les régions industrielles traditionnelles des nouveaux Länder, les possibilités de surmonter la crise structurelle provoquée par le passage à l'économie sociale de marché doivent être très différemment évaluées.

2 Après une décennie on peut dresser un bilan provisoire des différentes stratégies de développement économique régional. Généralement, on peut reconnaître dans les vieilles régions industrielles d'Allemagne orientale différents signes d'un changement structurel durable qui s'ordonne suivant deux directions principales :

-A. D'une part, on investit des milliards dans de grands projets en pensant qu'ils vont susciter d'autres investissements et déclencher des changements structurels régionaux.

- B. D'autre part, on soutient des structures de dimensions relativement petites comme celles qui existent notamment dans les régions où prédominent les villes petites et moyennes.

Le but principal du développement économique et spatial est toujours l'aide aux investissements à caractère durable. On entend par durable, le changement structurel qui prend en compte des objectifs économiques et socioculturels autant que des questions écologiques et encourage la recherche de solutions à ces problèmes. 


\section{La rupture des structures économiques après la réunification}

4 Dans sa configuration actuelle le Land de Saxe a été constitué le 3 octobre 1990. Il compte environ 4,5 millions d'habitants (1998) pour une superficie de $18413 \mathrm{~km}^{2}$, ce qui représente $5,5 \%$ de la surface et $5,2 \%$ de la population de la République fédérale d'Allemagne. La densité de $248 \mathrm{hab} . / \mathrm{km}^{2}$ est supérieure à la densité moyenne de l'Allemagne qui atteint $229 \mathrm{hab} . / \mathrm{km}^{2}$. La répartition de la population est très inégale. Une grande partie de la population se concentre dans des aires de fortes densités contrastant avec des espaces ruraux qui, en comparaison, sont peu densément peuplés. Malgré les changements économiques des dix dernières années, les structures fondamentales de répartition de la population ont été conservées (fig. 1).

Figure 1 : Les espaces économiques de la Saxe

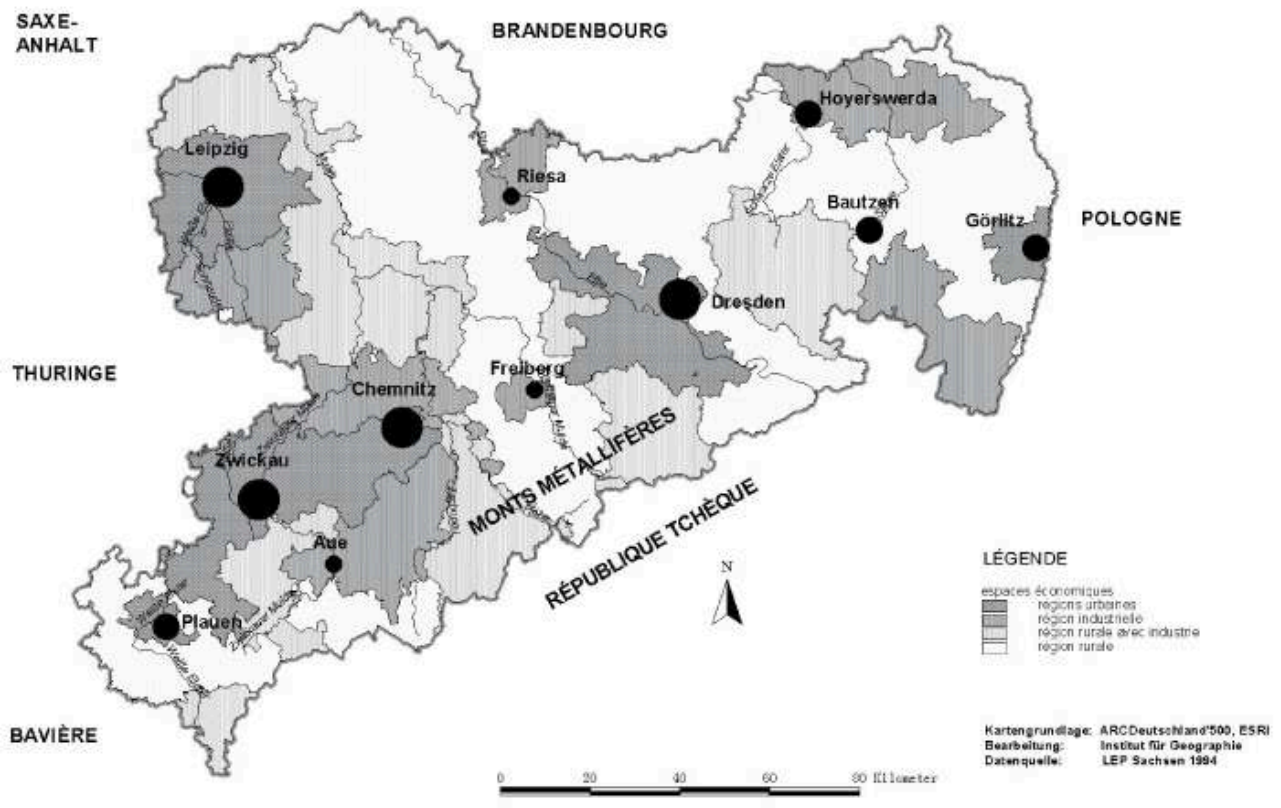

5 Le Land de Saxe est divisé administrativement en trois Regierungsbezirke. Près de $45 \%$ des frontières de la Saxe (environ $570 \mathrm{~km}$ ) sont en même temps des frontières externes de l'Union européenne. Les grandes différences de prix des denrées alimentaires et des services entre la Pologne, la République tchèque et la Saxe ont, au cours des dernières années, fortement désavantagé l'économie des régions saxonnes frontalières.

6 Au début du siècle, la Saxe faisait partie des plus importantes régions industrielles d'Allemagne et d'Europe. L'extraction des minerais et du lignite ainsi que la construction de machines et l'industrie textile comptaient parmi les branches industrielles les plus importantes. Après la seconde guerre mondiale, le poids de l'industrie dans l'ensemble de l'économie s'est conservé et a même été renforcé par la gestion économique centralisée de l'ancienne RDA. En comparaison des principaux états industrialisés occidentaux, la région industrielle saxonne présentait, lors de la réunification de 1990, un secteur industriel largement surdimensionné et un secteur tertiaire fortement sous-développé en ce qui concerne la structure des emplois. Ainsi, 
dans le cadre de l'actuel Land de Saxe, il y avait en 1990 encore 54 \% des actifs employés dans l'industrie et seulement $38 \%$ dans le secteur des services.

7 L'introduction des conditions du marché après la reconstitution de l'unité allemande a enclenché un difficile processus de transformation qui a abouti, au début des années 90 à une rupture structurelle caractérisée par les traits suivants :

- substitution de l'économie sociale de marché à l'économie planifiée centralisée,

- reconstitution d'ensemble de la propriété privée, c'est à dire reprivatisation de la propriété socialiste aussi bien dans l'agriculture que dans l'activité manufacturière (mines, industrie, commerce, etc) et dans le domaine personnel,

- reconquête de la liberté individuelle avec tous les droits et les devoirs d'un citoyen fédéral.

Ces changements structurels dans l'économie et la société qui, à cause de leur rapidité, ont pris un cours radical dans les domaines politique, intellectuel et socio-économique, ont été rendus plus difficiles par les facteurs suivants :

- des infrastructures obsolètes et insuffisantes,

- des procédés de production traditionnels déterminant une faible productivité du travail,

- la rupture avec les marchés d'Europe orientale,

- une insuffisante compétitivité et une mauvaise adaptation de l'économie au marché mondial,

- l'absence de management d'entreprise,

- le traitement bureaucratique par la Treuhand (ainsi que par son successeur « l'institut fédéral pour les charges spéciales nées de l'unification », le BvS) des conditions de la propriété suivant le principe « restitution plutôt que dédommagement ».

Dans le cadre des nouvelles conditions politiques et économiques, cette multitude de facteurs a freiné l'essor économique et provoqué de profonds changements sur le marché du travail. La diminution des actifs apparaît sur le tableau 1. En ce qui concerne les chiffres, il faut noter que, de 1989 à 1991, un million d'actifs ont été licenciés à la suite de l'arrêt total de l'extraction de l'étain et de l'uranium (Wismut), de la fermeture partielle du secteur de l'extraction du lignite, de la production d'énergie, de l'industrie chimique et de la fermeture d'une partie de l'industrie de transformation. En raison de leur fort taux d'activité, les femmes ont été particulièrement touchées par le chômage. Depuis le minimum de 1993 où le plancher a été atteint on assiste à un rétablissement inégal suivant les branches. Cependant, l'essor de nouvelles entreprises ne suffit pas, et de loin, à compenser les pertes d'emplois. Les mesures sociales, comme par exemple les « sociétés d'emplois » qui avaient d'abord été conçues comme des mesures transitoires, doivent être prolongées.

Tableau 1 : Nombre d'actifs par secteur d'activités de 1991 à 1998

\begin{tabular}{|l|c|r|r|c|}
\hline Secteur économique & \multicolumn{1}{|c|}{1991} & \multicolumn{1}{c|}{1993} & \multicolumn{1}{c|}{1998} & Recul en \% 1991-1998 \\
\hline Primaire & 101337 & 57716 & 53111 & 52 \\
Secondaire & 1008706 & 701931 & 715247 & 71 \\
Tertiaire & 1083139 & 1104519 & 1167134 & 111 \\
\hline Total & 2193182 & 1864166 & 1935492 & 88,2 \\
\hline
\end{tabular}

Source : Statistisches Landesamt des Freistaates Sachsen 1999, Kamenz.

10 Cette désindustrialisation doit être considérée comme un processus nécessaire pour effectuer la modernisation qui n'a pas été engagée en RDA au cours des années 80 . Ce 
processus a de toute façon été largement organisé de l'extérieur, c'est-à-dire depuis l'Allemagne de l'Ouest par l'intermédiaire de la politique économique. L'aide de l'état a été sans aucun doute sollicitée, le capital investi dans les transferts de l'Ouest vers l'Est, entre autres dans le cadre du programme «Essor de l'Est » ou encore dans le «Plan sur les transports Unité allemande" a été considérable. En Saxe, ce sont les sociétés d'assainissement des mines qui ont un poids particulièrement important. Des milliards d'investissements de l'état fédéral ont déjà été utilisés pour l'assainissement des anciens sites d'extraction de l'uranium et du lignite et on a pour le moment préservé 6000 emplois. Les différentes branches économiques et les régions de Saxe sont inégalement frappées par les mutations en cours. En 1989 le Land comptait 58 actifs pour 100 habitants; en 1996 ils n'étaient plus que 41.

11 Le recul de l'emploi, différencié dans le temps et dans l'espace, s'explique par les évolutions sectorielles inégales et par un grand nombre de fermetures d'entreprises. Ainsi, la diminution des actifs entre 1989 et 1996 est due pour $85 \%$ au secteur secondaire qui a perdu 835000 emplois et pour $15 \%$ au secteur primaire (tableau 1). Dans le secteur des services par contre, on a retrouvé en 1996 le niveau du passé. L'augmentation du nombre d'emplois est due à celle des services aux entreprises, notamment des instituts de crédit, des agences d'assurance et d'autres services nouvellement développés. Par contre, le nombre d'employés dans les services domestiques a fortement diminué.

12 La meilleure situation de l'emploi dans les régions urbaines est exclusivement due à la croissance du secteur tertiaire et s'est établie malgré une diminution supérieure à la moyenne des emplois industriels. Les grands besoins de rattrapage et l'incitation financière à la restauration des bâtiments ont provoqué un boom provisoire dans le secteur du bâtiment. Les légères améliorations de la situation de l'emploi enregistrées au cours des dernières années dans les espaces ruraux s'expliquent par ce que l'on peut, le cas échéant, considérer comme un effet de rattrapage du nombre d'employés dans les secteurs du commerce, des transports et de la diffusion de l'information ainsi que dans celui des services aux entreprises. La croissance d'ensemble, rapide et importante, des emplois dans le secteur des services, n'a pourtant pas pu compenser les pertes d'emplois, non seulement en volume mais surtout aux mêmes emplacements. Cela concerne en particulier les nombreux sites monoindustriels de Saxe comme la région de l'acier à Riesa, les bassins de lignite situés en Lusace et au sud de Leipzig, l'extraction de l'étain et de l'uranium dans les Monts métallifères. La dernière mine d'étain à Altenberg, où le minerai était exploité depuis 1440, a été fermée en 1991. Les régions textiles de Haute-Lusace, du piémont et de l'ouest des Monts métallifères ainsi que du Vogtland se sont réduites à quelques sites. Dans beaucoup de localités le déclin industriel a pour corollaires la dégradation de la situation sociale et l'émigration. Certaines régions n'ont plus aucune perspective d'un nouveau développement de l'emploi.

13 En avril 2000 le nombre des chômeurs en Saxe est descendu pour la première fois depuis des années en dessous des 400 000, ce qui représente $17,4 \%$ des actifs potentiels (par comparaison le taux de chômage en Bavière est de 5,5\%, 7,3\% en RhénaniePalatinat). Le plus fort taux de chômage en Saxe est celui du Bezirk de Bautzen en Haute-Lusace où il atteint $20,3 \%$.

Lors des dernières années, les mesures de retour à l'emploi ont fortement diminué, comme le montre l'exemple suivant : à la fin d'avril 2000, à Leipzig, il n'y avait plus que 
6530 actifs bénéficiant de mesures de retour à l'emploi alors qu'en 1992 ils étaient plus de 25000 . La conséquence de l'effondrement massif de l'emploi ainsi que des différences de niveau des salaires est la poursuite du courant d'émigration observable depuis 1989 et qui conduit les jeunes gens et les travailleurs qualifiés vers les anciens Länder. Cette évolution a pour conséquence de créer de nouvelles disparités régionales avec les conséquences démographiques et sociales correspondantes qui seront décrites plus précisément dans le paragraphe suivant.

\section{Les disparités régionales actuelles de développement}

Si l'on veut mettre en relief les disparités régionales il faut prendre en compte des indicateurs quantitatifs autant que qualitatifs. Les indicateurs importants pour la politique régionale, ce sont en particulier les différences de revenus et d'emplois, les caractéristiques des infrastructures ainsi que, de manière croissante, les caractéristiques démographiques et les formes d'utilisation de l'espace.

\section{A. L'évolution spatiale de la population}

De 1989 à 1998 le Land de Saxe a accusé une perte de population de plus de 300000 habitants (fig. 2). Comme pour tous les nouveaux Länder, l'essentiel de la diminution de la population en Saxe s'est produite au cours des années 1989/90 par émigration en direction des anciens Länder. Au total, au cours de ces deux années, environ 784000 personnes ont quitté le territoire de la RDA pour aller en Allemagne de l'Ouest. La plus grande partie d'entre elles, soit 402840 personnes, venaient de Saxe. En contrepartie il n'y a eu que 179930 immigrants. Ce sont principalement de jeunes travailleurs qualifiés à la recherche d'un travail bien rémunéré ainsi que de jeunes familles voulant se refaire une nouvelle existence dans les anciens Länder qui sont partis. Les destinations principales de la vague d'émigration étaient la Bavière, le Bade-Wurtemberg et la Rhénanie du Nord-Westphalie qui étaient en même temps les sources principales d'émigration vers la Saxe. Dès 1992 et 1993 les départs ont fortement diminué. La vague d'émigration s'est ainsi atténuée plus rapidement que ce que la plupart des observateurs prévoyaient. Malgré la permanence du bilan négatif, les statistiques de l'année 2000 attestent d'un solde migratoire positif avec les anciens Länder, le gain migratoire étant principalement dû à l'apport d'étrangers. 
Figure 2 : Évolution des migrations de population en Saxe

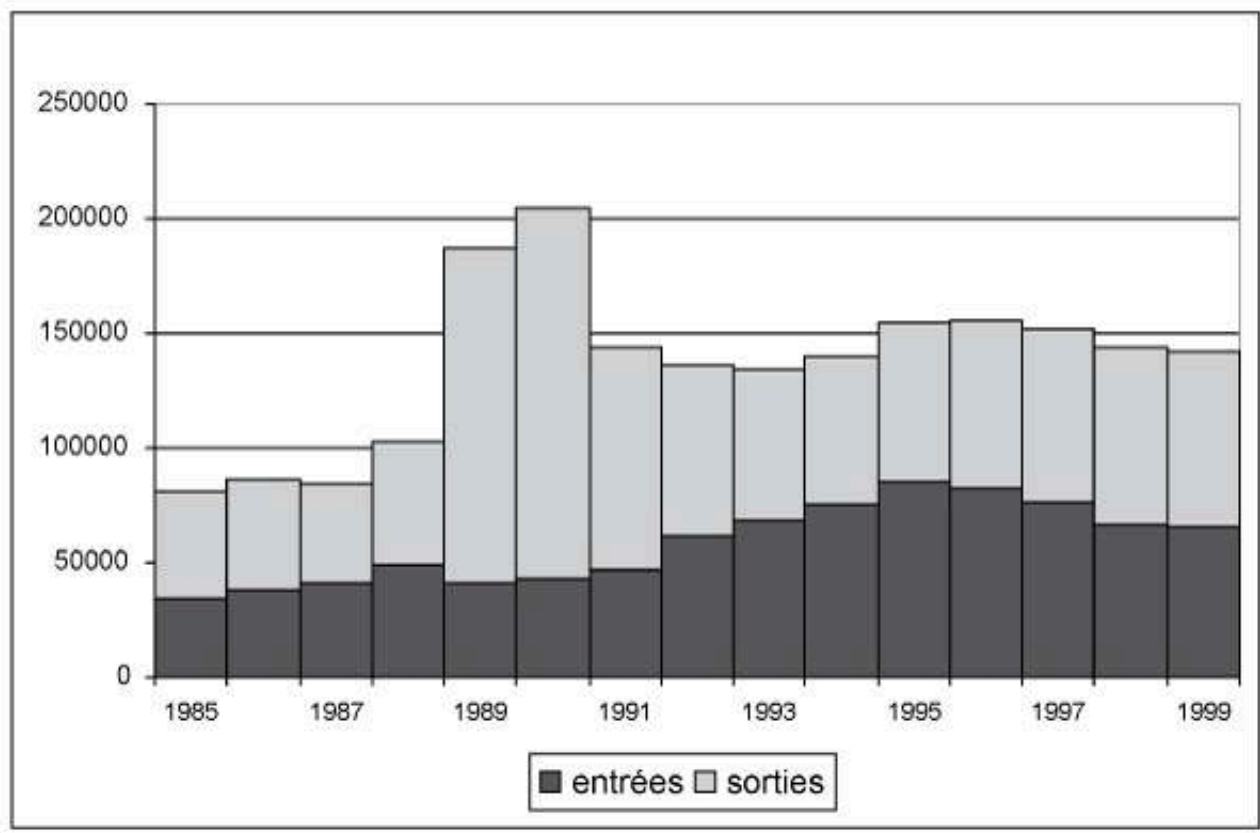

\section{B. Évolution naturelle de la population en Saxe}

Depuis 1989 les taux de natalité ont fortement diminué dans toutes les régions de Saxe. Il y a eu un recul très brutal de 1990 à 1991 lorsque le nombre de naissances pour 1000 habitants s'est réduit de $37,2 \%$ en moyenne. En ce qui concerne l'évolution des naissances, le plancher n'a été atteint qu'en 1994 (fig. 3). Depuis 1990 il n'y a plus aucun Kreis de Saxe qui présente une évolution naturelle positive, c'est-à-dire avec un excédent des naissances. Si l'on ne prend en compte que l'évolution naturelle de la population, la diminution en Saxe se chiffre à 205115 personnes de 1990 à 1996. Cela veut dire que, durant cette période, le nombre de naissances a été inférieur de 200000 à celui des décès. Mis à part le vieillissement de la population il faut y voir aussi la conséquence de l'émigration, puisque la grande masse des migrants des années 1989 et 1990 appartiennent à la génération des parents qui auraient pu, dans les années à venir, déterminer une augmentation du nombre des naissances. 
Figure 3 : Evolution naturelle de la population en Saxe de 1970 à 1998

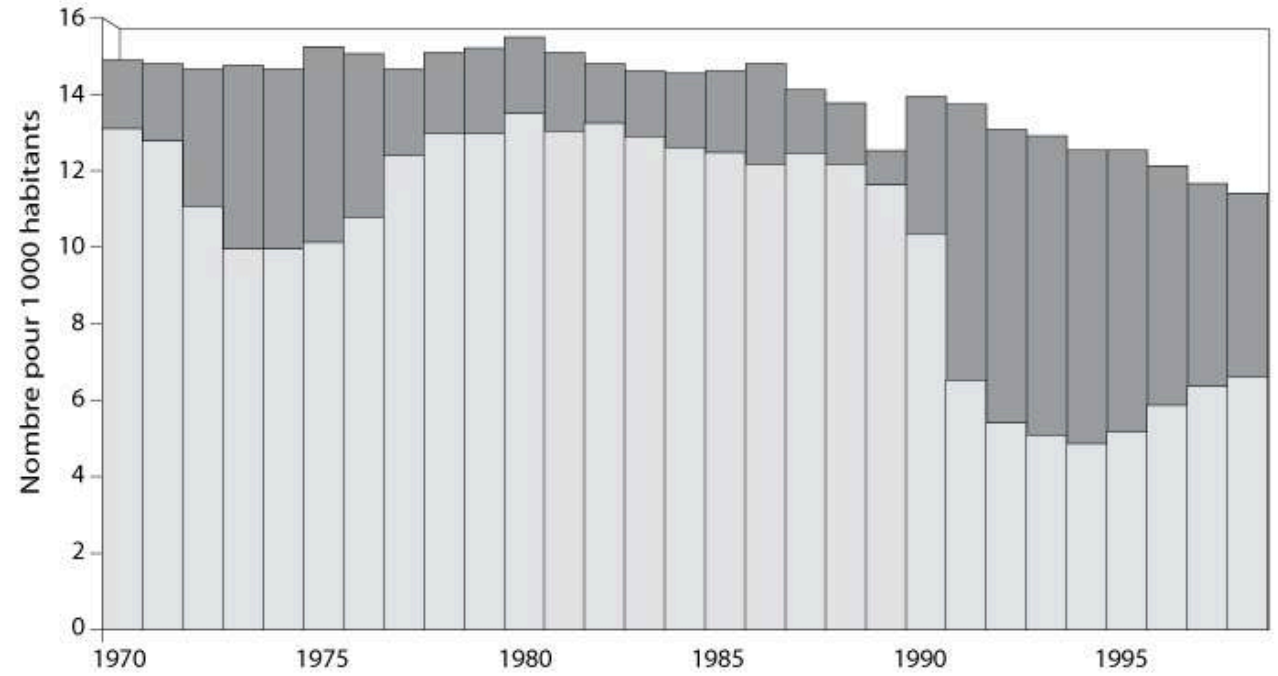

$\square$ Taux de natalité pour 1000 habitants $\square$ Taux de mortalité pour 1000 habitants

Les grandes pertes de migrants dans les classes d'âge les plus jeunes et le recul brutal du nombre de naissances après 1990, ont accentué encore le vieillissement de la population saxonne, dont la structure par âge en 1990 était déjà défavorable par rapport à celle des autres Länder. Un quart des habitants de la Saxe sont déjà à l'âge de la retraite (plus de 60 ans). Or, ce processus ne semble pas près de se terminer. Les prévisions établies par le service statistique du Land de Saxe pour la période 1995-2010 (tableau 2) tablent sur un alignement du comportement démographique de la population saxonne sur celui des anciens Länder. Cela signifie que, pour la période allant jusqu'en 2010, il faut s'attendre à une poursuite du déficit des naissances, même si dans presque tous les Kreis celui-ci se réduira. La différence entre la natalité et la mortalité va faire que la population saxonne va continuer à diminuer au cours des prochaines années, quoique de façon atténuée.

Tableau 2 : Prévisions d'évolution de la population par région

\begin{tabular}{|l|r|r|r|r|r|r|}
\hline & \multicolumn{2}{|c|}{1990} & \multicolumn{2}{c|}{2000} & \multicolumn{2}{c|}{2010} \\
\hline Planungsregion & absolu & $\%$ & absolu & $\%$ & \multicolumn{1}{c|}{ absolu } & $\%$ \\
\hline Oberlausitz, Niederschlesien & 721025 & 100 & 626003 & 86,8 & 617087 & 85,6 \\
Oberes Elbtal, Osterzgebirge & 1109505 & 100 & 1038298 & 93,6 & 1077611 & 97,1 \\
Chemnitz, Oberes Erzgebirge & 994696 & 100 & 904760 & 91,0 & 924946 & 93,0 \\
Westerzgebirge, Vogtland & 777427 & 100 & 715337 & 92,0 & 744029 & 95,7 \\
Westssachsen & 1161648 & 100 & 1066013 & 91,8 & 1092175 & 94,0 \\
Sachsen & 4764301 & 100 & 4350411 & 91,3 & 4455848 & 93,5 \\
\hline
\end{tabular}

Source : Amt für Statistik, Kamenz 1998.

\section{La périurbanisation à la périphérie des grandes villes saxonnes}

Le tournant politique en Allemagne orientale a provoqué un changement complet où la périurbanisation s'est substituée à l'urbanisation, le développement compact des villes cédant la place à une extension spatiale décentralisée dans les régions urbaines. Cette évolution a reposé sur la privatisation rapide du marché foncier et immobilier. En 
raison des conditions favorables d'amortissement offertes aux investisseurs, le capital immobilier national et international a influencé fortement l'évolution. La formidable périurbanisation amorcée en 1990 s'est caractérisée par un dynamisme particulier. Dans la première moitié des années 1990 elle dépassait déjà celle des anciens Länder lors de la phase culminante de périurbanisation des années 60 et du début des années 70. C'était en premier lieu une conséquence des transferts financiers et des possibilités fiscales d'amortissement pour la construction de maisons individuelles dans les nouveaux Länder.

Dans les régions urbaines de l'ex-RDA, avant 1990, il n'y avait pas de périurbanisation et les grandes villes (en tant que point de concentration de l'habitat) contrôlaient même les campagnes environnantes. Dans les grands ensembles d'habitat collectif, il n'y avait pas de formes de ségrégation économique. Les mécanismes d'attribution du logement conduisaient pourtant à un afflux sélectif de catégories de populations jeunes et, par là même, à une concentration de personnes à haute qualification professionnelle dans les grands ensembles situés pour la plupart en périphérie.

21 Après le tournant politique, la périurbanisation autour des grandes villes saxonnes s'est développée « auf der grünen Wiese » (littéralement sur la prairie), là où des sociétés de développement ouest-allemandes, profitant de l'absence temporaire de planification régionale, ont aménagé de nouveaux lotissements, en spéculant sur la possibilité de rentabiliser au maximum leurs investissements. Les projets ont pu y être réalisés plus rapidement et à meilleur compte que dans la ville. On ne peut qu'établir un parallèle avec les USA, beaucoup plus net dans les nouveaux Länder qu'en Allemagne de l'Ouest. L'évolution des régions urbaines des grandes villes saxonnes, influencée par les possibilités d'amortissement, a conduit, en peu de temps, à la multiplication des appartements et des immeubles de bureaux vides, ainsi qu'à celle des zones d'activités nouvellement aménagées mais inoccupées. Tous ces indices indiquent une saturation du marché de l'immobilier. Tandis que la périurbanisation du commerce de détail et des activités diminuait de nouveau au milieu des années 90, la périurbanisation de l'habitat a atteint son maximum en 1996, puis a reculé fortement avec la diminution des possibilités fiscales d'amortissement (voir Wießner, en ce volume). La caractéristique de la périurbanisation dans les nouveaux Länder est que, par nature, il s'agit d'une évolution induite par les possibilités d'amortissement fiscal.

Tandis que la périurbanisation de l'habitat se développe principalement aux dépens des grandes villes, la périurbanisation du commerce de détail et des activités a été alimentée principalement de manière exogène. Il s'agit essentiellement de filiales d'entreprises issues des anciens Länder (voir Hoppmann et Schmidt en ce volume). La perte de fonctions de production et de services dans les grandes villes saxonnes est ainsi moins le résultat de la périurbanisation que la conséquence de la rupture structurelle.

23 La forme qu'a prise la périurbanisation en Saxe correspond à un processus de déconcentration dans le contexte d'une diminution de l'emploi et de la population dans les régions des grandes villes. Ainsi, la périurbanisation de l'habitat en Saxe se différencie de façon significative des modèles de développement des régions urbaines en Europe occidentale. Typique de cette évolution est la déconcentration de la population dans les régions des grandes villes alors qu'en même temps la population diminue et, d'après les prévisions les plus récentes, continuera à diminuer. La forte émigration dans les campagnes environnantes constitue pour les villes-centres un tournant 
durable puisque, pour la deuxième fois depuis le changement politique, elles subissent d'importantes pertes d'habitants. La ville de Leipzig, par exemple, a perdu plus de 110000 habitants entre 1988 (545 307 habitants) et 1998, la diminution de la population à partir de 1993 se faisant essentiellement par émigration dans l'espace périurbain et les campagnes environnantes. Les régions entourant les grandes villes, au contraire, connaissent, pour la première fois depuis des décennies, une augmentation de leur population. Cette croissance résulte, non seulement de la venue de familles des classes moyennes-supérieures, les nouveaux propriétaires typiques des maisons individuelles des alentours des villes, mais aussi de l'arrivée de ménages aisés sans enfants (environ $15 \%$ de célibataires), dans des parcs résidentiels constitués de petits collectifs développés grâce aux possibilités de déduction fiscale. La périurbanisation de l'habitat autour des grandes villes, Dresde, Leipzig et Chemnitz, est ainsi entrée en concurrence avec la gentrification dans les villes-centres.

Les nouvelles structures d'habitat des alentours des grandes villes, liées le plus souvent à la circulation individuelle motorisée, se sont développées tantôt de façon dispersée, tantôt de façon linéaire le long des axes de circulation. L'extension spatiale très dynamique aux alentours des grandes villes saxonnes est aujourd'hui fortement atténuée. Les causes en sont la diminution de la demande et les surplus de zones d'activités et de zones commerciales. Même la périurbanisation de l'habitat est revenue à un taux normal, avec la réduction de moitié, depuis 1997, des déductions fiscales pour les nouvelles constructions.

\section{Potentiel de développement et grands investissements en Saxe}

Le Land de Saxe dispose d'un ensemble de potentialités significatives pouvant permettre un renouveau fondamental et une poursuite du développement de l'économie dans toutes les régions du Land. Parmi celles-ci, il s'agit entre autres : d'un réservoir de main-d'œuvre qualifiée; d'un réseau de communication dense; d'infrastructures de recherche et de formation de qualité ; d'une structure industrielle diversifiée, pouvant servir de base au développement de petites et moyennes industries; de longues traditions industrielles; de liens étroits avec les marchés d'Europe orientale; de ressources naturelles (lignite et matériaux de construction), ainsi que d'une offre d'activités culturelles diversifiées et de haute qualité.

Lors du remodelage des espaces industriels au cours des dernières années, on a aménagé de nouveaux grands sites qui, entre-temps, sont devenus parmi les plus modernes d'Europe et ont apporté une impulsion décisive aux régions environnantes. La microélectronique et l'industrie automobile ainsi que leurs sous-traitants comptent parmi les branches industrielles qui se sont le plus vite et le mieux développées en Saxe. À titre d'exemple, deux de ces grands investissements et leurs effets sur le marché régional du travail vont être maintenant examinés.

\section{A. Le développement de l'industrie automobile en Saxe}

L'usine Volkswagen (VW) de Mosel près de Zwickau constitue, pour parler comme le président de la Saxe, Biedenkopf, le phare d'une nouvelle industrie en plein essor en Allemagne orientale. Depuis le changement politique, Volkswagen a investi 3,5 
milliards de DM sur les sites de Chemnitz et de Mosel près de Zwickau. Actuellement, l'usine VW de Chemnitz produit environ 2000 moteurs par jour. Ils équipent entre autres la Polo et sont livrés également à la filiale espagnole de VW, Seat. Depuis 1996, on fabrique sur le site de Mosel, la Passat qui a succédé à la Polo. L'usine est aujourd'hui l'une des plus rentables du groupe. La millionième VW a quitté l'usine à la fin de l'année 1999. La Gmbh VW Sachsen est devenue l'entreprise au plus gros chiffre d'affaire d'Allemagne orientale (en 1999 le groupe VW Sachsen avait un chiffre d'affaire de 6,9 milliards de DM) et, avec un effectif de 7000 personnes, elle est l'un des plus gros employeurs de Saxe. Au cours de l'année 2000, le Konzern va investir 200 millions de DM supplémentaires sur son site saxon et il prépare la fabrication de véhicules à quatre roues motrices (Passat). À la fin de l'année, la carrosserie de la nouvelle automobile de luxe, la D1, sortira des chaînes de Mosel. Le véhicule sera ensuite assemblé dans la nouvelle « usine en verre » de Dresde. Les éléments décisifs pour le choix du nouveau site de Mosel à Zwickau ont été : la mise à disposition de subventions pour les investisseurs dans les Länder de l'Est, une main-d'œuvre qualifiée (90\% de travailleurs qualifiés), la bonne liaison du site avec la voie ferrée et l'autoroute, l'offre de terrains à bâtir, la demande du marché pour la VW Golf et surtout la présence d'entreprises de sous-traitance de l'industrie automobile, ainsi que l'expérience du travail industriel dans la région de Chemnitz-Zwickau.

Depuis 1990 une importante industrie de sous-traitance s'est également constituée. Plus de 35000 emplois dépendent de l'usine. La fabrication en juste à temps (just in time) qui lie la plupart des sous-traitants avec VW a été l'occasion, pour une multitude de petites et moyennes entreprises de la région, fabriquant depuis plus d'un siècle des pièces automobiles, de mettre en place une organisation plus moderne du travail et d'investir elles-mêmes dans de nouvelles techniques. La possibilité de livrer les produits juste à temps repose sur le faible éloignement des sous-traitants, ce qui permet d'éviter un stockage coûteux. 170 entreprises situées à proximité de l'usine sont intégrées dans cette organisation. Il y a maintenant en Saxe environ 320 entreprises privées dans l'industrie automobile. Depuis le début des années 90 environ 5 milliards de DM par an ont été investis dans la sous-traitance automobile, non seulement pour construire de nouvelles usines, mais aussi pour la recherche et le développement et cette évolution semble loin d'être terminée. Après avoir étudié 21 sites européens, le groupe automobile Toyota Europe a finalement décidé d'installer une nouvelle usine à Zwickau. Le 7 février 2000, la première pierre de la nouvelle usine Porsche a été posée sur le site de Leipzig (investissement : 100 millions de DM, 260 emplois, production de voitures tout-terrain). Ainsi, à côté du traditionnel triangle de l'automobile ZwickauChemnitz-Mosel, la Saxe a obtenu deux nouveaux grands sites à Dresde et Leipzig. Par là même la Saxe est progressivement en train de rattraper, en ce qui concerne la production automobile, les sites traditionnels de Bavière et du Bade-Wurtemberg. Les changements se sont accomplis à un rythme formidable. L'industrie automobile représente déjà $42 \%$ du chiffre d'affaire de l'industrie en Saxe; elle emploie au total 62000 personnes et sa productivité a déjà dépassé celle des sites d'Europe occidentale. Cependant, son insertion dans les circuits de production internationaux reste encore insuffisante. Le gros investissement que représente l'usine de montage VW de Mosel a ainsi imprimé une impulsion décisive pour le redémarrage économique de toute une région. 


\section{B. Le développement de la micro-électronique sur le site de Dresde}

\section{recherche des universités technologiques de Dresde et de Chemnitz), l'accueil très} favorable réservé au projet aussi bien par la population que par les décideurs.

31 Au temps de la RDA, Dresde était déjà un site important pour les semi-conducteurs et les équipements électroniques, ainsi qu'un site de recherche. Aussi, il existe, dans la région, une acceptation du travail à toute heure de la journée. La fabrication de puces électroniques nécessite en effet une organisation du travail en équipes se relayant sur $24 \mathrm{~h}$. employés, cela représente un investissement d'environ 1 million de DM par poste de travail, ce qui apparait nettement au-dessus de la moyenne des entreprises de l'industrie de transformation. Le projet a reçu environ 1 milliard de DM de subventions, ce qui représente à peu près un tiers des sommes totales investies $(0,35$ million de DM par poste de travail). D'après les prévisions actuelles, les investissements sur le site de Dresde auront, en l'espace de 10 ans, jusqu'en 2003/2004, dépassé 3 milliards de DM. Jusqu'à la fin de l'année 1999, 2,6 milliards de DM ont déjà été investis, dont 1,8 milliard pour les équipements.

Parmi les 2650 employés travaillant dans l'usine de semi-conducteurs, $81 \%$ viennent de Saxe, $5 \%$ d'autres nouveaux Länder, $13 \%$ des anciens Länder et $1 \%$ de l'étranger. D'autres emplois ont été créés dans le cadre de l'aménagement du site, 1121 emplois dans le secteur du bâtiment et encore 430 emplois dans le domaine des équipements. L'entreprise est aujourd'hui agrandie d'une nouvelle usine de puces électroniques dont la première pierre a été posée le 30 mai 2000. La filiale de Siemens, Infineon, projette d'investir encore 2 milliards de DM dans la première usine du monde où l'on fabriquera des puces à mémoire avec la nouvelle technologie des $300 \mathrm{~mm}$ (la production de pièces de silicium de $300 \mathrm{~mm}$ sert de base à la production de puces d'ordinateurs). 1100 emplois de plus doivent être ainsi créés. Ce qui a joué en faveur de Dresde comme site d'implantation de la nouvelle fabrication, c'est de nouveau l'infrastructure existante, c'est-à-dire l'important savoir-faire technologique disponible sur le site. L'état fédéral et le Land ont versé environ 350 millions de DM de subvention pour la nouvelle usine. Le nouveau module de fabrication doit, à la fin de 2002, atteindre une capacité de production d'environ 6000 pièces de silicium par semaine.

D'après les chiffres fournis par le groupe, Siemens emploie actuellement 7900 personnes en Saxe, en comptant les apprentis. Celles-ci se répartissent entre trois sites de distribution et sept de fabrication, parmi lesquels la nouvelle fabrique de téléphones de Leipzig. Mise à part l'usine de puces Siemens, «Silicon Saxony " (par référence au

Revue Géographique de l'Est, vol. 41 / 3 | 2001 
site high-tech américain de la Silicon Valley) a également attiré à Dresde le groupe américain AMD (Advanced Micro Devices). Une nouvelle usine de puces électroniques pour la fabrication de microprocesseurs à haute vitesse a également été installée pour un investissement se montant à 3,2 milliards de DM; elle emploie déjà 1200 personnes et en emploiera 1800 en 2001. En juin 2000 elle a commencé à livrer à la clientèle la nouvelle génération de microprocesseurs Athlon. Elle produit notamment le processeur Athlon K7 (nom grec désignant le prix offert au vainqueur) qui est actuellement le microprocesseur en cuivre le plus rapide du monde. Un fait particulièrement important pour le site de Dresde est que un quart des employés actuels était au chômage. De plus, plusieurs centaines de petites et moyennes entreprises de sous-traitance en microélectronique se sont installées autour des deux grandes entreprises Siemens et AMD. Ainsi Dresde constitue dès maintenant un des sites de microélectronique les plus intéressants du monde.

Le groupe Siemens a également investi sur les sites de Leipzig et de Görlitz. L'usine de 100 millions de DM construite à Leipzig emploie 500 personnes. En comparaison des conditions de production antérieures aux USA l'entreprise est $30 \%$ plus productive. Elle fabrique des téléphones et des visiophones. Sur le site de Görlitz, 50 millions de DM ont été investis dans une usine de turbines. Siemens chiffre le volume de ses investissements dans les nouveaux Länder, depuis le changement politique à 3,2 milliards de DM. Sur les 189000 employés allemands de Siemens il y en a aujourd'hui 13000 dans l'est. D'autres investissements sont en discussion, par exemple un projet de cinq milliards de DM, pour une nouvelle usine de puces du fabricant taïwanais de semiconducteurs Mosel Vitelic, qui pourrait créer jusqu'à 2000 emplois sur le site de Leipzig.

La politique de « conservation et de développement des noyaux industriels » ouvre une voie décisive pour la réindustrialisation de la Saxe. Celle-ci a été permise par la mise à disposition de subventions et favorise les régions urbaines, tout en se faisant au détriment des aides à l'investissement dans le secteur des moyennes entreprises qui constituait l'originalité de la Saxe. Néanmoins, malgré le manque de capital propre, il y a, à côté des intérêts internationaux, beaucoup d'initiatives d'entrepreneurs locaux qui, avec de nouvelles idées, renouent partiellement avec de vieilles traditions. Les nombreux nouveaux sites d'activités dans l'ouest des Monts métallifères en constituent un exemple.

Ainsi, les contours d'une nouvelle structure de l'économie apparaissent progressivement. Dresde comme site de haute technologie, Zwickau, Chemnitz et maintenant aussi Leipzig avec l'industrie automobile. Même les anciens bassins de lignite sont de véritables pôles de croissance reposant sur la production énergétique et la chimie. Ces derniers sont cependant encore trop peu nombreux.

Aussi, il n'y a plus que deux douzaines d'entreprises en Allemagne orientale ayant plus de un milliard de DM de chiffre d'affaire. Les entreprises industrielles en Allemagne orientale ont en moyenne 80 employés alors que c'est le double en Allemagne occidentale. Heureusement, on peut observer une tendance à l'augmentation des investissements dans les moyennes entreprises d'Allemagne orientale. La proportion de firmes qui développent de nouvelles branches est même plus importante à l'Est qu'à l'Ouest. En particulier dans les secteurs traditionnels de la chimie, de la métallurgie et de l'électronique/ électrotechnique les entreprises de l'Est ont déjà acquis une position technologique dominante dans leur branche. À certains projets on a sans doute attribué 
un caractère emblématique. Ce succès est également dû aux importants investissements à long terme dans les infrastructures des régions urbaines de Dresde, Leipzig et Chemnitz.

\section{Les changements spatiaux et structurels actuels}

En ce qui concerne la qualité des conditions de vie, il existe, à l'intérieur du Land, des situations très différentes en fonction, d'une part de la situation dans l'espace et d'autre part de la répartition spatiale des dégâts environnementaux les plus importants. Ceux-ci apparaissant comme particulièrement négatifs; beaucoup de régions présentent aujourd'hui, un besoin particulier de développement, d'assainissement et d'aide financière (fig. 4). Parmi les régions ayant le plus de problèmes en ce qui concerne les besoins en subventions, il y a les régions frontalières et les régions des mines d'uranium et de lignite.

Figure 4 : Régions nécessitant des aides particulières au développement et à l'assainissement

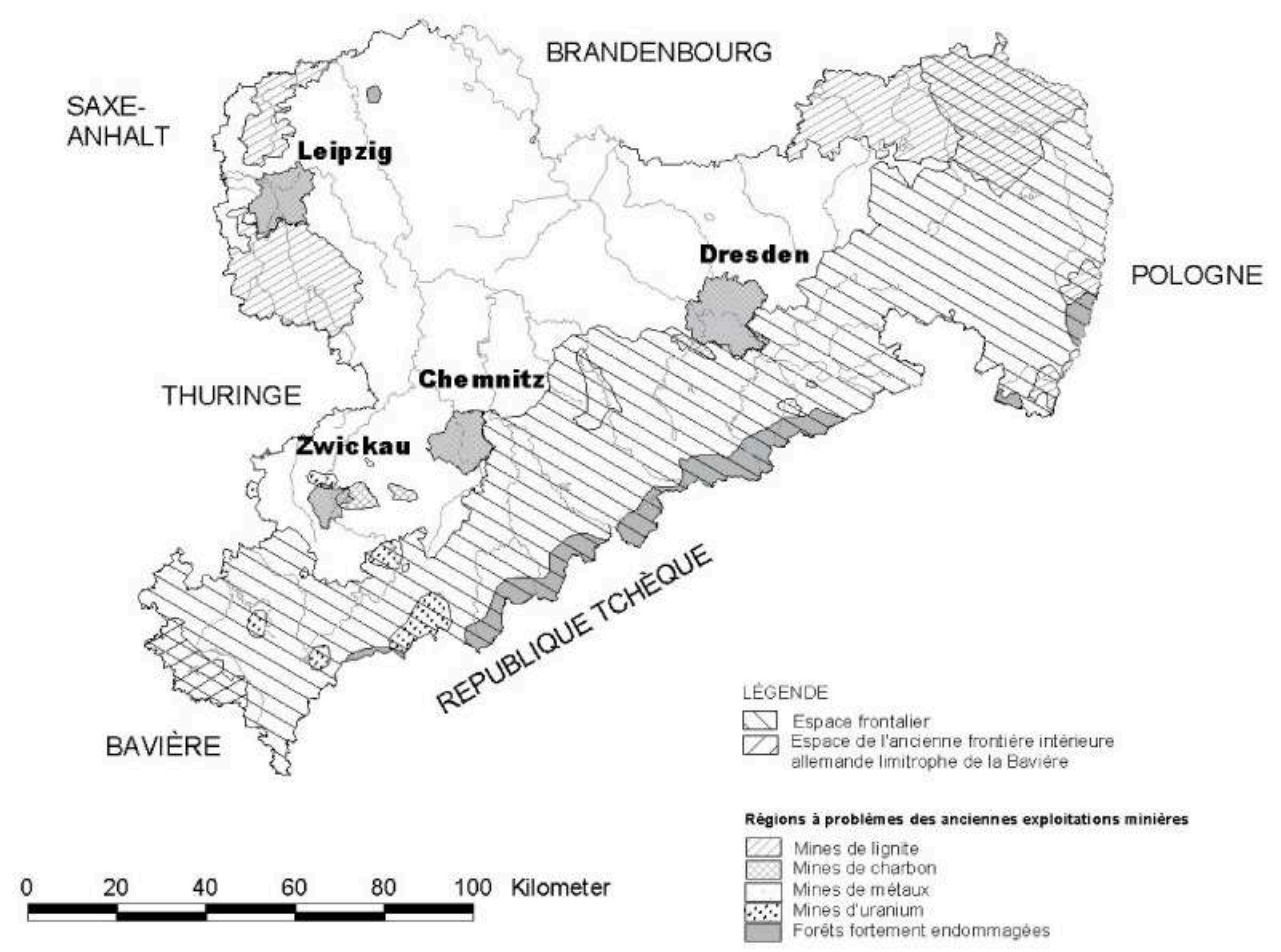

Aux régions à assainir et à aider financièrement il faut encore ajouter 10 espaces pour lesquels la planification du Land de Saxe a proposé des mesures et des concepts de développement régional (fig. 5). Il s'agit en l'occurrence d'espaces présentant des faiblesses structurelles et qui, dans le cadre de la collaboration intercommunale, ont adopté des concepts de développement décidés à l'échelle locale, notamment pour la réalisation d'infrastructures. Pour cela, ils reçoivent $\mathrm{du}$ Land le financement correspondant. Ainsi, par exemple, depuis le début des années 90, l'ancien bassin de lignite situé au sud de Leipzig a bénéficié de 200 millions de DM par an pour les travaux d'assainissement minier. Ces projets d'assainissement emploient actuellement environ 2000 personnes et vont être nécessaires pendant encore environ 10 ans. Le résultat des travaux d'assainissement des mines de lignite est le développement de nouveaux 
paysages artificiels, de qualité visuelle tout à fait convenable, qui représentent une chance décisive pour l'avenir en ce qui concerne les activités de détente, les loisirs dans la région, ainsi qu'en ce qui concerne l'attractivité pour l'habitat et la qualité de la vie.

Figure 5 : Zones d'application des mesures de développement régional depuis 1993

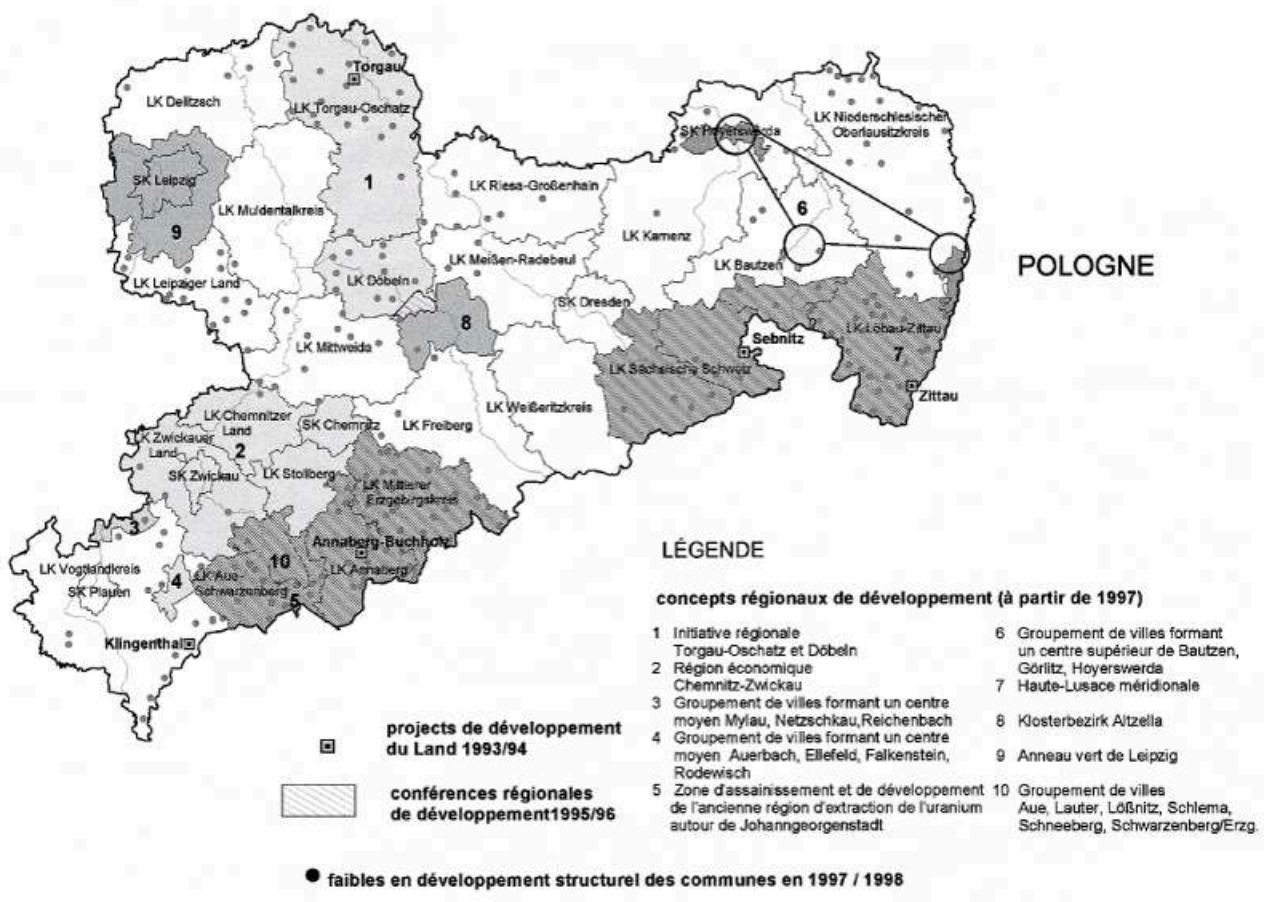

Le développement de la structure spatiale en Saxe a pour but d'élever progressivement le Land au niveau d'un important espace économique, scientifique et culturel à l'intérieur de l'Union européenne. Pour cela, ce qu'on appelle le "triangle de Saxe", constitué des centres supérieurs de Dresde, Leipzig et Chemnitz/ Zwickau (fig. 6) est appelé à jouer un rôle particulièrement important. Il doit être érigé en région urbaine européenne et, par là même, obtenir une position prioritaire dans le réseau urbain européen. Le concept de réseau de villes est issu des stratégies politiques européennes et il est caractérisé par le développement des relations fonctionnelles entre villes et communes. Les réseaux de villes sont aussi une stratégie alternative de diversification pouvant permettre de contrebalancer le gonflement démesuré d'un nombre limité de centres urbains européens. Le cadre directeur de la politique d'aménagement du territoire de la République fédérale s'inspire de ce concept en lui accordant une importance fondamentale. Les trois grandes villes saxonnes sont actuellement les supports principaux du développement économique en Saxe. Tout en étant en concurrence, elles ont également des intérêts communs. Ainsi, elles peuvent développer ensemble des stratégies de marketing et défendre leurs intérêts. La "Cityregion » européenne du "triangle de Saxe » doit avoir un rôle important comme centre de culture et de technologie pour le développement européen. Il s'agit d'un objectif stratégique à long terme de la planification du Land (30 à 50 ans). La compétitivité du Land de Saxe à l'intérieur de l'Europe doit être améliorée par un développement concerté des trois grandes villes, Dresde, Chemnitz et Leipzig. Cette "Cityregion du triangle de Saxe " constitue le point de départ essentiel pour la concentration des projets d'investissement dans le domaine des infrastructures. En ce 
qui concerne la circulation des personnes et des marchandises, il s'agit d'intégrer le triangle de Saxe dans le réseau européen à grande vitesse. Celui-ci doit être constitué de lignes ferroviaires rapides reliant les centres européens majeurs auxquels les régions périphériques seront rattachées par des liaisons autoroutières et aériennes. Les nœuds du triangle de Saxe doivent être ainsi intégrés dans les réseaux d'infrastructures nationales et transeuropéennes.

Figure 6 : Plan de développement du Land de Saxe : le triangle de Saxe

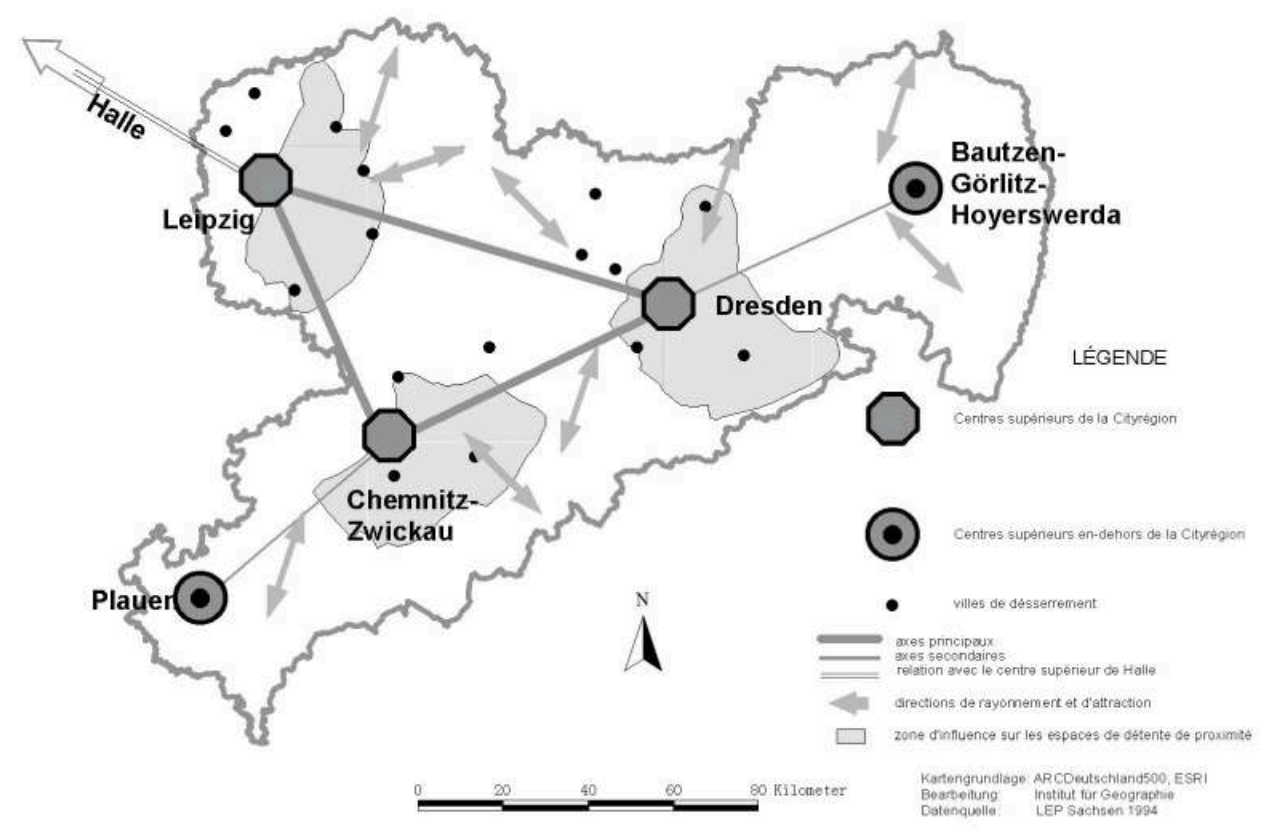

L'internationalisation de l'économie et de la société, accélérée par le marché intérieur européen, nécessite une coopération entre les pôles de développement à l'intérieur de la Saxe. Ce n'est qu'à cette condition que le potentiel interne pourra pleinement faire valoir les effets de synergie régionale et sera compétitif sur le plan international.

\section{Conclusion}

D'après une étude publiée en 1999 par un institut renommé de prévision de Bâle, la Saxe aura presque atteint, en 2020, le niveau économique de l'Ouest. Les économistes suisses tablent sur le fait que l'économie de l'Est atteindra bientôt des taux de croissance supérieurs à ceux des anciens Länder. Le taux de croissance était de $2,3 \%$ en 1998 (2,2 \% dans les nouveaux Länder, 2,8 \% dans les anciens Länder). Actuellement, l'Allemagne orientale a un produit intérieur brut qui atteint pratiquement $60 \% \mathrm{du}$ niveau de l'ouest. Dans 20 ans, d'après les prévisions, il devrait atteindre $90 \%$. À titre de comparaison, en 1991, la productivité économique de l'Est n'atteignait que 31,5\% de celle de l'Ouest. Cela montre l'importance du processus de rattrapage.

Le support de la croissance en Saxe est maintenant la grande industrie. Ainsi, le Konzern automobile BMW construira sa nouvelle usine à Leipzig et va ainsi investir 2 milliards de DM sur le nouveau site. L'usine et ses sous-traitants devraient permettre la création de près de 10000 emplois dans la région. On prévoit, pour la fin de l'année 2003, le démarrage de la fabrication du monospace qui, jusqu’à présent, est encore 
produit à Ratisbonne (Bavière). À l'origine, plus de 200 villes ou régions avaient fait acte de candidature pour l'installation du fabricant d'automobiles bavarois. Lorsque la décision a été prise, il restait encore en lice des villes comme Schwerin, Augsburg, Kolin en République tchèque et Arras en France. Ce qui a fait pencher la balance en faveur de Leipzig, c'est non seulement la qualité des infrastructures et de la main-d'œuvre, mais aussi le niveau des aides financières du Land et de l'état fédéral. Au total, en raison du nécessaire processus de rattrapage, les investissements effectués dans l'industrie estallemande depuis la réunification se sont élevées à 13,1 milliards de DM. En 2000, ces investissements se sont rapprochés d'un niveau «normal». Dans les années qui viennent, on s'attend à une forte diminution des investissements à l'est, même dans le cas d'une conjoncture favorable.

\section{BIBLIOGRAPHIE}

Blotevogel H.H. (1998). - Europäische Metropolregion Rhein-Ruhr. Theoretische, empirische und politische Perspektiven eines neuen raumordnungspolitischen Konzepts. Institut für Landesund Stadtentwicklungsforschung des Landes Nordrhein-Westfalen. Dortmund.

Bundesministerium für Raumordnung ; Bauwesen und Städtebau (1993). -

Raumordungspolitischer Orientierungsrahmen. Leitbilder für die räumliche Entwicklung der Bundesrepublik Deutschland. Bonn.

Herfert Günter (1994). - Suburbanisierung der Bevölkerung in Großstadtregionen Sachsens. In : Europa Regional 2. Leipzig. Heft 3.

Herfert Günter (1996). - Wohnsuburbanisierung in Verdichtungsräumen der Neuen Bundesländer. In : Europa Regional 4. Leipzig, Heft 1.

Sächsische Staatsregierung (1994). - Landesentwicklungsplan Sachsen vom 16. August 1994. Sächsisches Gesetz- und Verordnungsblatt, Nr. 51, Dresden.

Sächsische Landesanstalt für Landwirtschaft (1998). - Entwicklungsperspektiven für ländliche Räume. Dresden.

Schmidt Helga (1997). - Urbane Transformationsprozesse im Spiegel des Bodenmarktes der Stadt Leipzig. In : Mayr A. (Hrsg.) : Regionale Transformationsprozesse in Europa. Beiträge zur Regionalen Geographie 44. Leipzig.

Schmidt Helga (1999). - Potentiale im regionalen Kontext. Das Beispiel Südraum Leipzig. In : Zukunft Stadt-Land-Landschaft. Leipzig.

Schmidt Ralph (1997). - Wachstumsschwerpunkte und Disparitätenausgleich in Sachsen. In : Europa Regional 5. Leipzig. Heft 4.

Staatsministerium für Umwelt und Landesentwicklung Freistaat Sachsen (1995). Landesentwicklungsbericht 1994. Dresden.

Statistisches Landesamt des Freistaates Sachsen (1990-1999). - Statistisches Jahrbuch. Kamenz. 


\section{RÉSUMÉS}

Les 10 ans de la réunification de l'Allemagne constituent une bonne occasion de s'intéresser d'un point de vue géographique à la situation dans les nouveaux Länder. Les effets des changements structurels survenus durant les dix dernières années sont étudiés à partir de l'exemple du Land de Saxe. On peut en effet reconnaître des évolutions positives ou négatives résultant du processus de désindustrialisation. Malgré des différences régionales marquées, le taux de chômage reste, comme auparavant, supérieur à la moyenne des anciens Länder de la RFA. La spécificité des évolutions régionales est marquée par l'implantation d'entreprises dans les régions urbaines de Dresde, Leipzig et Chemnitz-Zwickau, favorisées par leur situation par rapport aux infrastructures de communication. La rupture des structures économiques a eu également des effets négatifs sur l'évolution démographique de la population saxonne. Tandis que l'émigration vers les anciens Länder de la RFA, prédominante après le tournant politique, s'est depuis lors affaiblie, le processus d'émigration de la population du centre des grandes villes saxonnes vers la périphérie s'est fortement renforcé. En raison de la persistance du recul de la population, il apparait de fortes différences régionales dans la structure socio-démographique du Land de Saxe. Il en résulte un accroissement des disparités régionales favorisant la croissance économique des régions urbaines au détriment des espaces périphériques situés le long des frontières avec la Pologne et la République tchèque. L'image de marque en partie négative des régions doit être changée par la modernisation de leurs infrastructures et l'amélioration du management régional (Le Triangle de Saxe).

In the course of German reunification the East German states, including the state of Saxony passed through a breathtaking transition process in all spheres of life. The major problem was the transformation of a comparatively inefficient industry-based and centrally governed economy into a modern market-based economy. The industrial state of Saxony recorded a tremendous number of factory closures and capacity reductions, leading to a rapid growth of

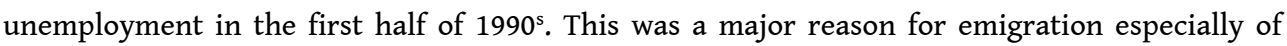
young and well educated persons. Hence Saxony's present and mid-term age structure appears quite unfavourable. The artificially-induced process of suburbanisation exacerbates the problems in the form of migration of wealthy families (valuable tax payers) from the core cities of agglomeration into the rural hinterland.

However, admirable efforts to deal with the structural crises by using the traditional endogeneous potentials of the Saxonian regions can be recognized. Through governmental schemes new investors could be attracted, in particular in the prosperous fields of car production, engineering and IT. The development of « industrial cores » also promotes the sector of medium and small-scale industry, though some lack of governmental assistance for these is apparent. Another sector gaining from the overall positive industrial development since the mid 1990's is the service sector. It provides more jobs than in the period before 1990. However, current efforts are not reflected in a significant change in the labour market. The inadequate support of largely mechanized industrial units is obviously not enough to tackle the basic problem of unemployment.

In order to enhance Saxony's competitiveness at European and Global levels the state planning authorities and scientists follow the strategy of regional cooperation. Based on the city region «Sachsendreieck» the comparative regional advantages will be used in order to offer a wide range of services for the demands of national, continental and global markets.

Despite serious problems Saxony has the potential to ensure, in the long run, a sustainable socioeconomic development as the outcome of a painful transitional process during the past decade. To achieve this goal the joint actions of Saxonian citizens, the political class and the Saxonian entrepreneurs are required. 
Zehn Jahre Vereinigung Deutschlands sind Anlass genug, sich aus geographischer Sicht mit der aktuellen Situation in den neuen Bundesländern $\mathrm{zu}$ beschäftigen. Die Auswirkungen des Strukturwandels der letzten zehn Jahre werden am Beispiel des Freistaates Sachsen behandelt. In Sachsen lassen sich sowohl positive als auch negative Tendenzen eines nachhaltigen Strukturwandels erkennen, der vor allem vom Prozess der Deindustrialisierung bestimmt wird. Die Arbeitslosenquote liegt in Sachsen bei ausgeprägten Unterschieden nach wie vor über dem Durchschnitt der alten Bundesländer. Spezifische regionale Entwicklungsmuster kristallisieren sich bei der Analyse der Standortansiedlung von Unternehmen zugunsten der verkehrsgünstig gelegenen Verdichtungsgebiete Dresden, Leipzig und Chemnitz-Zwickau heraus. Der wirtschaftliche Strukturbruch hat sich auch negativ auf die sächsische Bevölkerungsentwicklung ausgewirkt. Während die nach der politischen Wende dominierende Abwanderung der Bevölkerung in die alten Bundesländer zwischenzeitlich abgeflaut ist, hat sich seitdem der Prozess der Stadt-Randwanderung zuungusten der sächsischen Großstädte erheblich verstärkt. Aufgrund des insgesamt anhaltenden Bevölkerungsrückgangs ergeben sich innerhalb des Freistaates deutliche regionale Unterschiede hinsichtlich der sozialdemographischen Struktur ihrer Bewohner. Daraus resultiert wiederum ein zunehmender Anstieg regionaler Disparitäten, durch den das wirtschaftliche Wachstum der Verdichtungsräume auf Kosten der peripheren Räume entlang der Staatsgrenze zu Polen und Tscheschien begünstigt wird. Das teilweise negative Standortimage der Regionen soll durch den gezielten Ausbau ihrer Infrastruktur und die Verbesserung des Regionalmanagements (Sachsendreieck) verbessert werden.

\section{INDEX}

Keywords : city region, deindustrialisation, regional disparities, reunification, Saxony, suburbanisation, triangle of Saxony

Schlüsselwörter : Deindustrialisierung, regionale Disparitäten, Sachsen, Sachsendreieck, Suburbanisierung, Verdichtungsräume, Wiedervereinigung

Mots-clés : désindustrialisation, disparités régionales, périurbanisation, régions urbaines, réunification, Saxe, Triangle de Saxe

\section{AUTEUR}

\section{HELGA SCHMIDT}

Universität Leipzig - Institut für Geographie - Johannisallee 19a, 04103 Leipzig, Allemagne 